\title{
Prick and intradermal skin tests in patients with severe hymenoptera sting allergy using commercial versus in-house allergen extracts
}

\author{
Nualanong Visitsunthorn, ${ }^{1}$ Kittipos Visitsunthorn, ${ }^{2}$ Nitat Sookrung, ${ }^{3}$ Witchaya Srisuwatchari, ${ }^{1}$ \\ Punchama Pacharn, ${ }^{1}$ Orathai Jirapongsananuruk ${ }^{1}$
}

\begin{abstract}
Background: Fire ant, honey bee, and wasp allergen extracts are useful in the diagnosis and treatment of severe Hymenoptera allergic patients.

Objective: To evaluate the result of skin prick test (SPT) and intradermal test (ID) compared between local and commercial insect allergen extracts in patients with severe Hymenoptera sting allergy.

Methods: SPT and ID using local and commercial insect allergen extracts were performed. Specific $\operatorname{IgE}$ (sIgE) to honey bee, wasp, and fire ant; component-resolved diagnosis (CRD); (rApi m1, rApi m2, rApi m3, rApi m5, rApi m10, rVes $\mathrm{v} 5$, rPol d5, and rVes v1); and, cross-reactive carbohydrate determinant (CCD) were performed.

Results: Twenty-seven patients were included. Twenty-five had anaphylaxis, and 2 had severe systemic skin reaction. Positive skin test (SPT and/or ID) result from local and commercial allergen extracts was $74 \%$ vs. $67 \%$ for fire ant, $48 \%$ vs. $59 \%$ for honey bee, and $52 \%$ vs. $74 \%$ for yellowjacket. Local and commercial allergen extracts showed substantial agreement for fire ant $(k=0.647, p=0.001)$ and honey bee $(k=0.632, p=0.001)$, and moderate agreement for wasp ( $k=0.547, p=0.001)$. When compared with sIgE subtracted with CCD and/or CRD, skin test results of local fire ant allergen extract showed higher sensitivity ( $87 \% v$ s. $67 \%)$, specificity ( $42 \% v$ s. $33 \%)$, and accuracy $(67 \% v s .52 \%)$ than commercial extract. Commercial honey bee and wasp showed higher sensitivity (62\% vs. $50 \%, 85 \%$ vs. $65 \%)$ and accuracy $(63 \%$ vs. $52 \%, 78 \%$ vs. $70 \%)$, respectively.
\end{abstract}

Conclusion: SPT and ID with local or commercial insect venoms could help in confirming and/or identifying the causative insects.

Key words: Allergen extracts, Anaphylaxis, Component-resolved diagnosis, Insect allergy, Specific IgE to venom

From:

${ }^{1}$ Division of Allergy and Immunology, Department of Pediatrics,

Faculty of Medicine Siriraj Hospital, Mahidol University, Bangkok, Thailand

${ }^{2}$ Saint Louis University School of Medicine, Saint Louis, Missouri, USA

${ }^{3}$ Biomedical Research Incubator Unit, Department of Research, Faculty of Medicine Siriraj Hospital, Mahidol University, Bangkok, Thailand

Corresponding author:

Nualanong Visitsunthorn

Division of Allergy and Immunology, Department of Pediatrics

Faculty of Medicine Siriraj Hospital, Mahidol University

2 Wanglang Road, Bangkoknoi, Bangkok 10700, Thailand

E-mail: nualanongv@yahoo.com

\author{
Abbreviations/acronyms \\ SPT skin prick test \\ ID intradermal test \\ SIgE specific IgE \\ CRD component-resolved diagnosis \\ CCD cross-reactive carbohydrate determinant
}

\section{Introduction}

Severe allergic reaction from Hymenoptera insect sting is not uncommon. A study from Thailand found that $7.7 \%$ of anaphylaxis cases in the emergency room were caused by insect sting allergy. ${ }^{1}$ A retrospective study conducted at Siriraj Hospital (Bangkok, Thailand) reported that $11 \%$ of anaphylaxis cases were suspected to be insect-related. ${ }^{2}$ Determination of the causative insect is important for preventing future stings, 
and for treatment with immunotherapy. Sting history and clinical manifestations are useful in the diagnosis of Hymenoptera sting allergy, but identification of the causative insect is often quite difficult. To confirm the causative insect in severe allergic cases, although sting challenge tests considered being the gold standard in the diagnosis. However, it is not recommended as a routine diagnostic method, thus skin tests (skin prick test [SPT] and intradermal test [ID]) and/or the determination of specific immunoglobulin E (sIgE) to honey bee, wasp, hornet, and fire ant venoms should be performed.,

SPT and ID with insect venoms and/or quantification of sIgE to insect venoms and component-resolved diagnosis (CRD) are useful methods for identifying the causative insect. ${ }^{5}$ Skin test is easier, less expensive, and more sensitive ${ }^{6}$ than sIgE (RAST), but it cannot be performed shortly after severe allergic reaction, in patients currently taking antihistamines, or in patients with extensive skin lesions. Commercial allergen extracts are periodically unavailable. However, honey bee, common wasp, white-faced hornet, yellow hornet, and yellow-jacket venom extracts and fire ant whole body extract were commercially available at the time this study was conducted. These extracts are used not only for the diagnosis via skin testing, but also for specific treatment with immunotherapy. The aforementioned periodic lack of availability of commercially available allergen extracts can prevent diagnosis by skin test, and can interrupt the continuity of immunotherapy treatment. Improvement in the availability of venom extracts in our region is urgently needed.

To improve the availability of these allergen extracts, we developed extracts from the common stinging insect species in Thailand, ${ }^{7-9}$ including honey bee venom extract from Apis dorsata, wasp venom extract from Vespa affinis, and fire ant whole body extract from Solenopsis geminata. The purpose of developing these extracts is to ensure a reliable source for extracts in case commercial extracts are unavailable, and to reduce the cost of these extracts so that patients in Thailand and other countries in the region will have access to diagnosis of and treatment for insect allergy. However, the developed extracts must be tested both in vitro and in vivo to evaluate their efficacy and safety. After in vitro testing for safety and efficacy, in vivo evaluation must be performed to compare our locally-produced extracts with commercially available insect allergen extracts.

The purpose of this study was to evaluate the result of SPT and ID compared between local and commercial insect allergen extracts in patients with severe hymenoptera sting allergy, and to compare the results of SPT and ID of both local and commercial allergen extracts to the result of CRD.

\section{Materials and Methods}

The research protocol was approved by the Institutional Ethics Committee of the Faculty of Medicine Siriraj Hospital, Mahidol University, Bangkok, Thailand (COA no. 450/2559 [EC3]). This study was registered with ClinicalTrials.gov (NCT03645291). Written informed consent from parents or guardians and assent from children older than 7 years of age were obtained.

\section{Preparation of local insect allergen extracts}

Wasps (Vespa affinis) were collected from Nakhon Ratchasima province, which is located in lower Northeastern Thailand. Honey bees (Apis dorsata) and fire ants (Solenopsis geminata) were collected from Chiang Mai province, which is located in central Northern Thailand. Adult insects were verified by an entomologist. The freshly collected mature insects were kept frozen until allergen extract processing.

For fire ant whole body extraction, fire ants were ground by mortar and pestle, homogenized by sonication in PBS with $0.8 \%$ phenol, and then centrifuged. The supernatant was collected, filled with glycerol 1:2 v/v (50\% final concentration), and filtered with a 0.2 micron membrane filter. The fire ant whole body extract was maintained at $2-8^{\circ} \mathrm{C}$ until use.

For wasp and bee venom extraction, the venom sac was removed from each insect using forceps and scissors, after which it was washed with phosphate buffered saline (PBS) $\mathrm{pH}$ 7.4 and then placed into a small volume of buffer with $0.8 \%$ phenol. The venom was collected from each sac, pooled, and centrifuged at $12,000 \times \mathrm{g}$ for 20 minutes at $4^{\circ} \mathrm{C}$. The supernatant was collected and the protein concentration was measured. The venom solution was diluted to $200 \mu \mathrm{g} / \mathrm{ml}$ with PBS containing $0.8 \%$ phenol, filled with glycerol 1:2 v/v (50\% final concentration), and filtered using a membrane filter (pore size $0.2 \mu \mathrm{m})$. Wasp and bee venom extracts were maintained at $2-8^{\circ} \mathrm{C}$ until use.

Individual batch of the extracts was determined protein quantity and quality by Bradford assay and SDS-PAGE, respectively. Protein content of the extracts in each batch was standardized according to the commercial extract. Sterility testing was determined according to the medical device standards. In addition, skin testing to all local insect allergen extracts were performed in ten healthy control subjects and the results were all negative.

\section{Studied population}

This study in patients with severe allergic and anaphylactic reactions to stinging insects was performed at the Division of Allergy and Clinical Immunology, Department of Pediatrics, Faculty of Medicine Siriraj Hospital, Mahidol University, Bangkok, Thailand during the June 2018 to May 2019 study period. Severe allergic reaction was defined as a generalized reaction that could be life-threatening, such as generalized urticaria with angioedema. Anaphylaxis was defined as an acute severe reaction involving more than 1 anatomical system, including cutaneous, respiratory, circulatory, and/or gastrointestinal components. ${ }^{10}$

\section{Study methods}

Patient sting history was collected and recorded. SPT with local and commercial insect allergen extracts was performed in all patients. The commercial insect allergen extracts used in this study were fire ant (Solenopsis invicta) whole body extract (ALK-Abello, Port Washington, NY, USA), honey bee (Apis mellifera), wasp (Polistes fuscatus), white-faced hornet (Dolichovespula maculate), yellow hornet (Dolichovespula arenaria) and yellowjacket (Vespula vulgaris) venom extracts (Jubilant all 5 from HollisterStier LLC, Spokane, WA, USA). 
Table 1. Source and concentration of local and commercial insect allergen extracts.

\begin{tabular}{ccccc}
\multirow{2}{*}{ Allergenic Extract } & \multicolumn{2}{c}{ Local } & \multicolumn{2}{c}{ Commercial } \\
\cline { 2 - 5 } Whole body fire ant & Solenopsis geminata & $1: 10 \mathrm{w} / \mathrm{v}$ & Solenopsis invicta & $1: 100 \mathrm{w} / \mathrm{v}^{* *}$ \\
\cline { 2 - 5 } Honey bee venom & Apis dorsata & $100 \mu \mathrm{g} / \mathrm{ml}$ & Apis mellifera & $\begin{array}{c}120 \mu \mathrm{g} \mathrm{venom} \\
\text { protein } / 1.2 \mathrm{ml}{ }^{*}\end{array}$ \\
Yellow jacket venom & Vespa affinis & $100 \mu \mathrm{g} / \mathrm{ml}$ & Vespula vulgaris & $\begin{array}{c}120 \mu \mathrm{g} \mathrm{venom} \\
\text { protein } / 1.2 \mathrm{ml}^{*}\end{array}$ \\
\hline
\end{tabular}

* Jubilant HollisterStier LLC

** ALK-Abello, Inc.

Comparison of the source and concentration of local and commercial insect allergen extracts is shown in Table 1. Skin prick tests were performed by applying one drop of $1 \mu \mathrm{g} / \mathrm{mL}$ venom extract to the forearm, and then pricking the skin through the surface of the drop with a sterile lancet. Skin response was assessed after approximately 15-20 minutes. In patients who had negative SPT to $1 \mu \mathrm{g} / \mathrm{mL}$ concentration of insect allergen extract, ID for that insect allergen extract was performed starting at a concentration of $0.001 \mu \mathrm{g} / \mathrm{mL}$. A $1 \mathrm{~mL}$ tuberculin syringe with a short 27-gauge needle was used to deliver a volume of $0.05 \mathrm{~mL}$ for intradermal testing. The needle was introduced into the superficial layers of the skin, bevel down, until the bevel was completely buried. A $0.05 \mathrm{~mL}$ aliquot of the venom dilution was then slowly injected, which resulted in the development of a small bleb. If a negative skin reaction was obtained within 20 minutes, ID testing was continued using 10-fold increases in allergen extract concentration until a reaction consisting of a 5-10 mm wheal and 11-20 $\mathrm{mm}$ erythema was obtained, or until a concentration of $1 \mu \mathrm{g} /$ $\mathrm{mL}$ was tested - whichever occurred first. A patient should be considered sensitive to the test venom when a skin response of 5-10 $\mathrm{mm}$ wheal and 11-20 $\mathrm{mm}$ erythema or greater occurs at a concentration of $1 \mu \mathrm{g} / \mathrm{mL}$ or less. ${ }^{11}$

Specific IgE levels of fire ant (i70), honey bee (i1), yellowjacket (i3), and paper wasp (i77); component-resolved sIgE: rApi $\mathrm{m} 1$ (i208), rApi $\mathrm{m} 2$ (i214), rApi $\mathrm{m} 3$ (i215), rApi m5 (i216), rApi m10 (i217), rVes v5 (i209), rPol d5 (i210), and rVes v1 (i211); and, cross-reactive carbohydrate determinant (MUXF3 CCD, i214) were measured by ImmunoCap (UniCAP 100; Phadia AB, Uppsala, Sweden). Positive results of sIgE, CRD, and CCD were interpreted when the levels were $\geq$ $0.35 \mathrm{kUA} / \mathrm{L}$.

Fire ant was concluded to be the causative insect if fire ant sIgE -CCD was > $0.35 \mathrm{kUA} / \mathrm{L}$. Honey bee was concluded to be the causative insect if honey bee sIgE subtracted with CCD ( $\operatorname{sgE}-\mathrm{CCD}$ ) was $>0.35 \mathrm{kUA} / \mathrm{L}$, and/or if any of the honey bee CRDs (rApi m1, m2, m3, m5, or m10) was positive. Wasp was concluded to be the causative insect if wasp sIgE-CCD was > $0.35 \mathrm{kUA} / \mathrm{L}$, and/or if any of the wasp CRDs (rVes v5, rPol d5, or rVes v1) was positive.

Mastocytosis was clinically evaluated according to WHO classification. ${ }^{12}$ Baseline serum tryptase was assayed by ImmunoCap to rule out mastocytosis. Serum tryptase level exceeding $20 \mathrm{mcg} / \mathrm{L}$ is one of the WHO minor criteria for diagnosing systemic mastocytosis. ${ }^{12}$
A flow diagram of the study protocol is shown in Figure 1.

\begin{tabular}{|c|c|}
\hline \multicolumn{2}{|c|}{$\begin{array}{l}27 \text { patients with severe allergic reaction } \\
\text { (25 with anaphylaxis, } 2 \text { with severe cutaneous reaction) }\end{array}$} \\
\hline \multicolumn{2}{|c|}{$\begin{array}{l}\text { - History taking } \\
\text { slgE to honey bee, wasp, fire ant and component } \\
\text { resolved diagnosis (rApi m1, rApi m2, rApi m5 } \\
\text { and rApi m10, rVes v5, rPol d5, rVes v1 } \\
\text { and cross-reactive carbohydrate determinant) } \\
\text { - Serum tryptase } \\
\text { - Skin prick test (SPT) to local and commercial honey bee, } \\
\text { wasp and fire ant allergen extracts }\end{array}$} \\
\hline \multirow[t]{2}{*}{ SPT positive } & SPT negative \\
\hline & $\begin{array}{l}\text { Intradermal test with local } \\
\text { and commercial allergen } \\
\text { extracts that the patient } \\
\text { showed negative SPT }\end{array}$ \\
\hline
\end{tabular}

Figure 1. Flow of the participants in the study.

\section{Statistical analysis}

Patient demographic and clinical data are presented as number and percentage (\%), mean \pm standard deviation (SD), or median and range (minimum, maximum), as appropriate. The agreement of positive and negative results between local and commercial allergen extracts was evaluated using Cohen's kappa statistic. The kappa value was categorized as no agreement $(<0.00)$, none to slight (0.01-0.20), fair (0.21-0.40), moderate (0.41-0.60), substantial (0.61-0.80), and almost perfect agreement (0.81-1.00). ${ }^{13}$ Sensitivity, specificity, and accuracy were used to evaluate the validity of the results of SPT to local and commercial allergen extracts, using CRD or sIgE$\mathrm{CCD}$ as a reference standard test. Association between history of causative insects and the results of sIgE -CCD or CRD was analyzed by Fisher's exact test. A p-value less than 0.05 was considered statistically significant. All statistical analyses were performed using PASW Statistics (SPSS) 18.0 (SPSS, Inc., Chicago, IL, USA). 


\section{Results}

Twenty-seven patients with anaphylaxis or severe systemic skin reaction to Hymenoptera insects (fire ant, honey bee, wasp, and hornet) were included in this study. The mean age of patients was 13.8 years (range: 5-25). Anaphylaxis was diagnosed in 25 patients. The most common organ involvement in anaphylactic patients was skin (24/25 patients, 96\%). Respiratory symptom was the second most common (19/25 patients, 76\%) followed by circulatory symptoms (6/25 patients, $24 \%$ ) and gastrointestinal symptoms (4/25 patients, $16 \%$ ). Two patients with severe systemic skin reaction suffered from generalized urticaria with severe angioedema. More than $60 \%$ of patients developed symptoms within 15 minutes after being stung, and $93 \%$ developed symptoms within one hour after being stung. The demographic and clinical characteristics of study patients are presented in Table 2 . The causative insects by history were fire ant in 12/27 patients (44.4\%), honey bee in $9 / 27$ patients $(33.3 \%$ ), and wasp or hornet in $9 / 27$ patients (33.3\%) (Table 3). The duration from the last sting or bite was 5 years among all patients, and 3 years among $80 \%$ of patients. The most common location where the sting occurred was home, followed by school and garden. The most common part of the body to be stung was foot (37.4\%), followed by hand or finger (29.6\%). Seventy-eight percent of patients were admitted to the hospital at least once due to insect sting or bite.

Among all patients, SPT to local fire ant whole body allergen extract showed $63 \%$ positivity, and the commercial extract showed $55 \%$ positivity. SPT to local honey bee venom allergen extract showed $22 \%$ positivity, and the commercial version showed $33 \%$ positivity. SPT to local yellowjacket venom allergen extract showed $33 \%$ positivity, whereas commercial common wasp, white-faced hornet, yellow hornet, and yellowjacket showed positive $33 \%, 44 \%, 26 \%$, and $44 \%$, respectively.

The SPT result of local fire ant whole body allergen extract showed moderate agreement with commercial fire ant whole body allergen extract $(k=0.542, p=0.004)$. The SPT result of local honey bee venom allergen extract showed fair agreement with commercial honey bee venom allergen extract $(k=$ $0.364, p=0.049$ ). The SPT result of local yellowjacket venom allergen extract showed moderate agreement with commercial wasp venom allergen extract $(k=0.500, p=0.001)$ and yellow hornet venom allergen extract $(k=0.471, p=0.013)$, and showed substantial agreement with white-faced hornet $(k=$ $0.615, p=0.001$ ). Agreement between local yellowjacket venom allergen extract and commercial yellowjacket venom was fair $(k=0.308, p=0.100)$, as shown in Table 4a.

When the results of SPT and ID were combined (Table 3 and Table $4 \mathbf{b}$ ), the positivity of local allergen extract increased to $74 \%$ for fire ant, to $48 \%$ for honey bee, and to $52 \%$ for yellowjacket. The positivity of commercial allergen extract also increased to $67 \%$ for fire ant, to $59 \%$ for honey bee, and to $74 \%$ for the wasp group. Positive SPT or ID of any commercial extract of common wasp, white-faced hornet, yellow hornet, or yellowjacket was reported as a positive skin test (SPT + ID) result. Agreement analysis between local and commercial extracts showed significant agreement for fire ant SPT and ID
Table 2. Demographic data of studied population $(\mathbf{n}=27)$

\begin{tabular}{|c|c|}
\hline Clinical characteristic & n (\%) \\
\hline Mean age (years) $\pm S D$, range & $13.8 \pm 5.0,5-25$ \\
\hline Sex: Male & $16(59.2 \%)$ \\
\hline \multicolumn{2}{|l|}{ Symptoms of insect allergy } \\
\hline Anaphylaxis & $25(92.6 \%)$ \\
\hline Severe systemic skin reaction & $2(7.4 \%)$ \\
\hline \multicolumn{2}{|l|}{ Other allergic diseases* } \\
\hline Asthma & $5(18.5 \%)$ \\
\hline Allergic rhinitis & $13(48.1 \%)$ \\
\hline Allergic rhino-conjunctivitis & $4(14.8 \%)$ \\
\hline Atopic dermatitis & $1(3.7 \%)$ \\
\hline Drug allergy & $3(11.1 \%)$ \\
\hline Food allergy & $5(18.5 \%)$ \\
\hline Family history of allergy in first degree cousins & $10(37 \%)$ \\
\hline \multicolumn{2}{|l|}{ Causative insects by history ${ }^{*}$} \\
\hline Fire ants & $12(44.4 \%)$ \\
\hline Bee & $9(33.3 \%)$ \\
\hline Wasp and hornet & $9(33.3 \%)$ \\
\hline \multicolumn{2}{|l|}{ No. of stinging (Times) } \\
\hline $1-2$ & $22(81.5 \%)$ \\
\hline $3-4$ & $2(7.4 \%)$ \\
\hline $4-5$ & $2(7.4 \%)$ \\
\hline$>5$ & $1(3.7 \%)$ \\
\hline \multicolumn{2}{|l|}{ Last stinging before enrollment (Year) } \\
\hline $0-1$ & $8(29.6 \%)$ \\
\hline$>1-3$ & $14(51.9 \%)$ \\
\hline$>3-5$ & $5(18.5 \%)$ \\
\hline \multicolumn{2}{|l|}{ Time from stinging to symptom (minutes) } \\
\hline $0-15$ & $18(66.7 \%)$ \\
\hline$>15-30$ & $3(11.1 \%)$ \\
\hline$>30-60$ & $4(14.8 \%)$ \\
\hline$>60$ & $2(7.4 \%)$ \\
\hline Treatment with epinephrine injection & $26(96.3 \%)$ \\
\hline Prophylaxis with epinephrine auto-injected & $25(92.6 \%)$ \\
\hline Treatment with antihistamine & $24(88.9 \%)$ \\
\hline Treatment with systemic corticosteroid & $12(44.4 \%)$ \\
\hline
\end{tabular}

*Some patients have more than one diagnosis and causative insect by history 


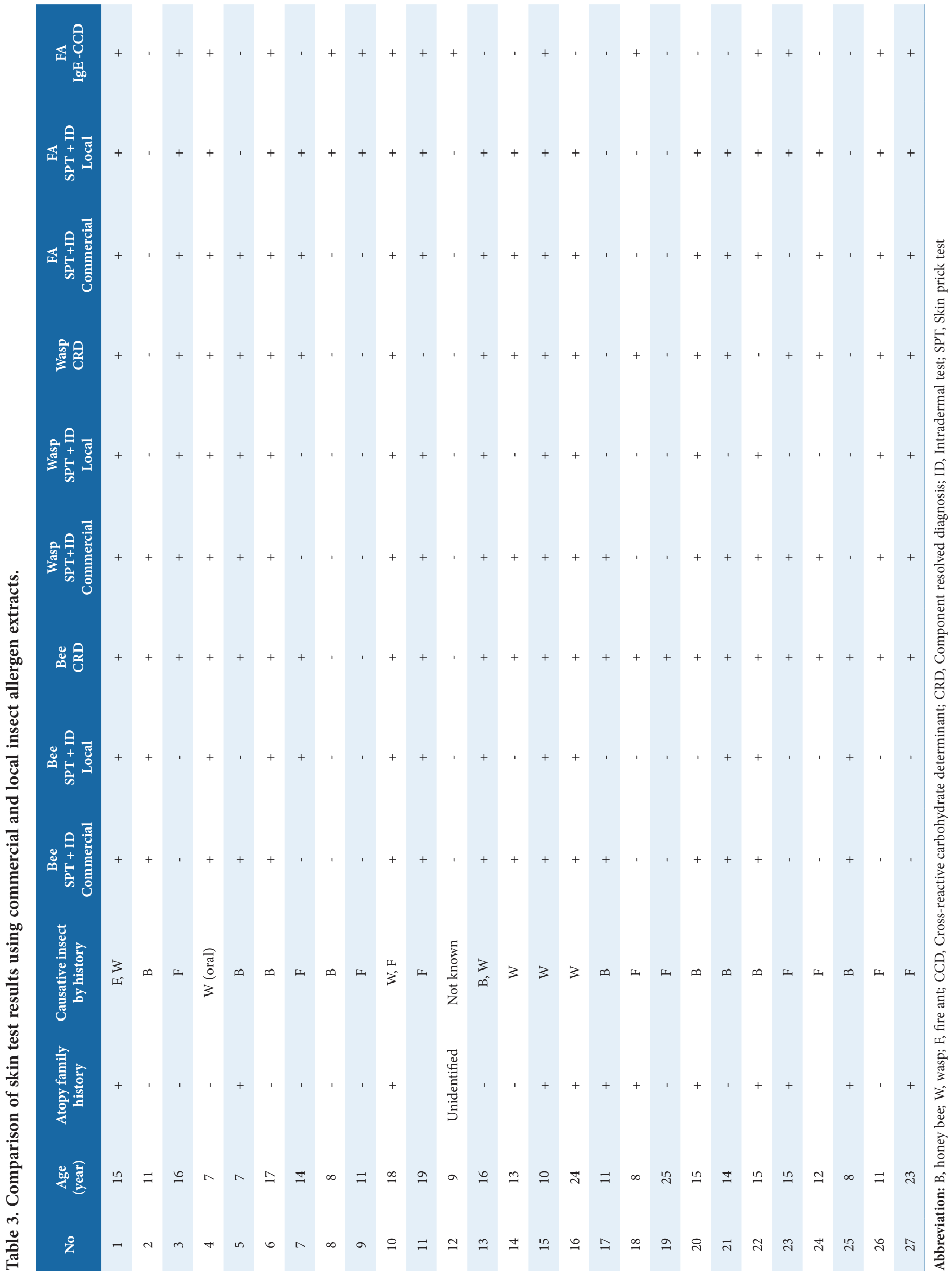


Table 4. Agreement analysis between commercial and local allergen extracts $(n=27)$

4a. Skin prick test result

\begin{tabular}{|c|c|c|c|c|}
\hline \multirow{2}{*}{ Commercial } & \multicolumn{2}{|c|}{ Local } & \multirow{2}{*}{$p$-value } & \multirow{2}{*}{ Kappa } \\
\hline & Negative & Positive & & \\
\hline Fire ant & & & 0.542 & 0.004 \\
\hline Negative & $8(30 \%)$ & $4(15 \%)$ & & \\
\hline Positive & $2(7 \%)$ & $13(48 \%)$ & & \\
\hline Bee & & & 0.364 & 0.049 \\
\hline Negative & $16(59 \%)$ & $2(7 \%)$ & & \\
\hline Positive & $5(19 \%)$ & $4(15 \%)$ & & \\
\hline Common wasp & & & 0.500 & 0.009 \\
\hline Negative & $15(56 \%)$ & $3(11 \%)$ & & \\
\hline Positive & $3(11 \%)$ & $6(22 \%)$ & & \\
\hline White- faced hornet & & & 0.615 & 0.001 \\
\hline Negative & $14(52 \%)$ & $1(4 \%)$ & & \\
\hline Positive & $4(15 \%)$ & $8(29 \%)$ & & \\
\hline Yellow hornet & & & 0.471 & 0.013 \\
\hline Negative & $16(59 \%)$ & $4(15 \%)$ & & \\
\hline Positive & $2(7 \%)$ & $5(19 \%)$ & & \\
\hline Yellow jacket & & & 0.308 & 0.100 \\
\hline Negative & $12(45 \%)$ & $3(11 \%)$ & & \\
\hline Positive & $6(22 \%)$ & $6(22 \%)$ & & \\
\hline
\end{tabular}

$(k=0.647, p=0.001)$ and honey bee SPT and ID $(k=0.632$, $p=0.001)$, and moderate agreement for wasp SPT and ID $(k$ $=0.547, p=0.001$ ).

When SPT and ID were compared with sIgE subtracted with CCD (sIgE-CCD) and/or CRD (as gold standard), we found that ID increased the sensitivity and accuracy of SPT to fire ant, honey bee, and wasp, but not specificity. The validity of SPT and ID of local and commercial fire ant, honey bee, and wasp allergen extract when compared with sIgE-CCD of fire ant and sIgE-CCD or CRD of honey bee and wasp 4b. Skin prick test combined with intradermal test result

\begin{tabular}{|c|c|c|c|c|}
\hline \multirow{2}{*}{ Commercial $^{*}$} & \multicolumn{2}{|c|}{ Local } & \multirow{2}{*}{$p$-value } & \multirow{2}{*}{ Kappa } \\
\hline & Negative & Positive & & \\
\hline Fire ant & & & 0.001 & 0.647 \\
\hline Negative & $6(22 \%)$ & $3(11 \%)$ & & \\
\hline Positive & $1(4 \%)$ & $17(63 \%)$ & & \\
\hline Bee & & & 0.001 & 0.632 \\
\hline Negative & $10(37 \%)$ & $1(4 \%)$ & & \\
\hline Positive & $4(15 \%)$ & $12(44 \%)$ & & \\
\hline Wasp & & & 0.001 & 0.547 \\
\hline Negative & $7(26 \%)$ & $0(0 \%)$ & & \\
\hline Positive & $6(22 \%)$ & $14(52 \%)$ & & \\
\hline
\end{tabular}

${ }^{*}$ Commercial wasp skin prick test combined with intradermal test results from Table $4 \mathrm{~b}$ were the combination of the results of wasp, white- faced hornet, yellow hornet and yellow jacket as a gold standard is shown in Tables $\mathbf{5 a}, \mathbf{5 b}$, and $\mathbf{5 c}$, respectively. Positive SPT or ID of any commercial extract of common wasp, white-faced hornet, yellow hornet, or yellowjacket was reported as a positive skin test (SPT + ID) result. Combined SPT and ID result of local fire ant whole body extract showed higher sensitivity (87\% vs. 67\%), specificity ( $42 \%$ vs. $33 \%$ ), and accuracy (67\% vs. $52 \%$ ) than commercial fire ant whole body extract. History alone showed 64\% sensitivity, 70\% specificity, and 67\% accuracy when compared with fire ant sIgE-CCD as a gold standard. Combined SPT

Table 5. Validity of skin prick test and intradermal test of local and commercial insect allergen extract when compared with specific immunoglobulin $\mathrm{E}$ and/or component-resolved diagnosis as gold standard

5a. Fire ant whole body allergen extract

\begin{tabular}{lccc} 
& \multicolumn{3}{c}{ sIgE-CCD (Gold standard) } \\
\cline { 2 - 4 } & Sensitivity & Specificity & Accuracy \\
\hline SPT (C) & $8 / 15(53 \%)$ & $5 / 12(42 \%)$ & $13 / 27(48 \%)$ \\
SPT + ID (C) & $10 / 15(67 \%)$ & $4 / 12(33 \%)$ & $14 / 27(52 \%)$ \\
SPT (L) & $11 / 15(73 \%)$ & $6 / 12(50 \%)$ & $17 / 27(63 \%)$ \\
SPT + ID (L) & $13 / 15(87 \%)$ & $5 / 12(42 \%)$ & $18 / 27(67 \%)$ \\
History alone & $9 / 14(64 \%)$ & $7 / 10(70 \%)$ & $16 / 24(67 \%)$ \\
\hline
\end{tabular}


5b. Bee venom allergen extracts

\begin{tabular}{lcccccc} 
& \multicolumn{2}{c}{ Sensitivity } & \multicolumn{2}{c}{ Specificity } & \multicolumn{2}{c}{ Accuracy } \\
\cline { 2 - 7 } Gold standard & CRD & $\begin{array}{c}\text { IgE-CCD or } \\
\text { CRD }\end{array}$ & CRD & $\begin{array}{c}\text { IgE-CCD or } \\
\text { CRD }\end{array}$ & CRD & $\begin{array}{c}\text { IgE-CCD or } \\
\text { CRD }\end{array}$ \\
\cline { 2 - 7 } SPT (C) & $9 / 24(38 \%)$ & $9 / 26(35 \%)$ & $3 / 3(100 \%)$ & $1 / 1(100 \%)$ & $12 / 27(44 \%)$ & $10 / 27(37 \%)$ \\
SPT + ID (C) & $16 / 24(67 \%)$ & $16 / 26(62 \%)$ & $3 / 3(100 \%)$ & $1 / 1(100 \%)$ & $19 / 27(70 \%)$ & $17 / 27(63 \%)$ \\
SPT (L) & $6 / 24(25 \%)$ & $6 / 26(23 \%)$ & $3 / 3(100 \%)$ & $1 / 1(100 \%)$ & $9 / 27(33 \%)$ & $7 / 27(26 \%)$ \\
SPT + ID (L) & $13 / 24(54 \%)$ & $13 / 26(50 \%)$ & $3 / 3(100 \%)$ & $1 / 1(100 \%)$ & $16 / 27(59 \%)$ & $14 / 27(52 \%)$ \\
History alone & $8 / 21(38 \%)$ & $9 / 21(39 \%)$ & $2 / 3(67 \%)$ & $1 / 1(100 \%)$ & $10 / 24(42 \%)$ & $10 / 24(42 \%)$ \\
\hline
\end{tabular}

\section{5c. Wasp venom allergen extracts}

\begin{tabular}{lcccccc} 
& \multicolumn{2}{c}{ Sensitivity } & \multicolumn{2}{c}{ Specificity } & \multicolumn{2}{c}{ Accuracy } \\
\cline { 2 - 6 } Gold standard & CRD & $\begin{array}{c}\text { IgE-CCD or } \\
\text { CRD }\end{array}$ & CRD & $\begin{array}{c}\text { IgE-CCD or } \\
\text { CRD }\end{array}$ & CRD & $\begin{array}{c}\text { IgE-CCD or } \\
\text { CRD }\end{array}$ \\
\cline { 2 - 6 } SPT (C) & $9 / 18(50 \%)$ & $9 / 20(45 \%)$ & $9 / 9(100 \%)$ & $7 / 7(100 \%)$ & $18 / 27(67 \%)$ & $16 / 27(59 \%)$ \\
SPT + ID (C)* & $16 / 18(89 \%)$ & $17 / 20(85 \%)$ & $5 / 9(56 \%)$ & $4 / 7(57 \%)$ & $21 / 27(78 \%)$ & $21 / 27(78 \%)$ \\
SPT (L) & $9 / 18(50 \%)$ & $9 / 20(45 \%)$ & $9 / 9(100 \%)$ & $7 / 7(100 \%)$ & $18 / 27(67 \%)$ & $16 / 27(59 \%)$ \\
SPT + ID (L) & $12 / 18(67 \%)$ & $13 / 20(65 \%)$ & $7 / 9(78 \%)$ & $6 / 7(86 \%)$ & $19 / 27(70 \%)$ & $19 / 27(70 \%)$ \\
History alone & $5 / 17(29 \%)$ & $7 / 19(37 \%)$ & $6 / 8(75 \%)$ & $6 / 6(100 \%)$ & $11 / 25(44 \%)$ & $13 / 25(52 \%)$ \\
\hline
\end{tabular}

${ }^{*}$ Commercial wasp skin prick test combined with intradermal test results from Table $5 c$ were the combination of the results of wasp, white- faced hornet, yellow hornet and yellow jacket

Abbreviations: C, commercial insect allergen extract; CRD, component-resolved diagnosis; ID, intradermal test; L, local insect allergen extract; SPT, skin prick test, sIgE-CCD, specific immunoglobulin E subtracted with cross-reactive

and ID result of commercial honey bee venom extract showed higher sensitivity (62\% vs. 50\%) and accuracy (63\% vs. 52\%) than local honey bee venom extract with equal specificity (100\%) when compared with sIgE-CCD and/or CRD of honey bee as a gold standard. The result of bee and wasp skin tests compared with only CRD as gold standard was similar to when compared with both sIgE-CCD and/or CRD as gold standard. History alone showed low sensitivity and accuracy, but high specificity. The combined SPT and ID result of commercial wasp venom extract showed higher sensitivity (85\% vs. $65 \%)$ and accuracy $(78 \%$ vs. $70 \%)$ than local honey bee venom extract, but lower specificity (57\% vs. $86 \%)$ when compared with sIgE-CCD or CRD of wasp as a gold standard. When compared with only CRD, the results were similar. History alone showed low sensitivity, but high specificity.

No systemic reaction from SPT or ID was observed when using either local or commercial insect allergen extracts. The only side effect was local swelling, pain, and/or itching, which was mild and improved with antihistamine. Serum tryptase level was less than $6 \mu \mathrm{g} / \mathrm{L}$ in all studied patients.

\section{Discussion}

This study showed that SPT and ID with local and commercial insect allergen extracts in patients with severe insect allergic reaction were comparable in sensitivity and accuracy, and that they showed significant agreement. In patients with negative SPT, the additional ID gave the skin test higher sensitivity and accuracy in patients with fire ant, honey bee, or wasp allergy.
SPT positivity was found in more than $50 \%$ of patients with severe fire ant allergic reaction, but in less than $40 \%$ of patients with severe honey bee and wasp allergic reactions. Positivity of SPT with local and commercial yellowjacket venom allergen extract was $33 \%$ and $44 \%$, respectively. The higher rate of the positive SPT results to fire ant than when using only the clinical history might due to the fact that there is some in vitro cross-reactivity between bee, wasp, and fire ant. Patients with bee, and wasp allergy could also have a positive SPT results to fire ant. However, when using sIgE-CCD as a gold standard to diagnose fire ant allergy, the sensitivity increase, but lower in specificity when using SPT and ID, as compared to those with history alone. In contrast, for patients with bee, or wasp allergy, double positive sensitization is not uncommon. Therefore, diagnosis of those insect allergy cannot be done by using only the clinical history.

Differences in the species of insects used in the preparation of extracts might be the cause of differences in skin test results. Skin test with local allergen extracts should be preferred over commercial versions since the insects used in commercial extracts may not be the same species as the insects in other areas. Ideally, local insect allergen extracts should be prepared from the common local insects that are the common cause of insect allergy in that area. In our study, Solenopsis geminata was used instead of Solenopsis invicta for preparing fire ant whole body allergen extract; Apis dorsata was used instead of Apis mellifera for preparing bee venom allergen extract; and, Vespa affinis was used instead of Vespula vulgaris for preparing yellowjacket venom allergen extract. 
These species of insects are the common causative insects for insect sting allergy in Thailand. ${ }^{7-9}$ The venom allergens of different honey bee species are highly similar and highly cross-reactive. ${ }^{14}$ Cross-reactivity between Polistinae and Vespinae venoms (especially yellowjacket venom) is frequently observed. ${ }^{15,16}$ Different ant venoms share some common protein components, but each group has a number of unique allergen components. ${ }^{17}$

Athough there were only 27 participants in this study, all of them had severe allergic reaction and 25 cases had anaphylaxis. By limiting the study population to only those with severe insect allergic reaction, we reduced the effect that variation in severity would have had on the test result. The high proportion of negative SPT to local and commercial honey bee and wasp venom allergen extracts $(78 \%, 66 \%, 67 \%$, and $56-74 \%$, respectively) may have been due to the younger age of patients in this study (mean age: 13.8 years. range: 5-25). Children had lower skin test reactivity to culprit venom, and lower ID sensitivity than adults. ${ }^{18}$ There were no differences between venom sIgE levels in relation to anaphylaxis grade, regardless of age. The mean level of bee venom sIgE was highest in the youngest children, with an observed subsequent decrease in sIgE up to age 30 years but there was no correlation between wasp venom sIgE and age. ${ }^{18}$

This study was performed within 5 years after the last sting, and $81 \%$ of our patients had their last sting within 3 years. This could affect the rate of positivity of sIgE and skin test, but it should not affect the comparison result since we compared skin test and sIgE in the same patients. Specific IgE was found to decrease during 1 to 4 years after Hymenoptera venom anaphylaxis, and it may fall below the level of detection with very long latency periods. ${ }^{5}$ The rate of loss of sensitization to Hymenoptera venom in skin tests was $12 \%$ per year, with $33 \%$ of skin tests becoming negative after 2.5 years. ${ }^{19}$ Negative skin test results and failure to detect venom sIgE in patients with a convincing history of Hymenoptera venom anaphylaxis may merely reflect a long latency period between sting event and diagnostic testing, ${ }^{18,19}$ especially in children since they have milder reactions and better recovery due to having a strong cardiovascular system. ${ }^{18}$ However, sensitization can remain detectable for many years in a number of patients.

To evaluate the sensitivity, specificity, and accuracy of skin test results from local and commercial insect allergen extract, we used CRD as a gold standard. Sting challenge with live insect is a gold standard in diagnosis of insect allergy, but it is too dangerous in anaphylactic insect sting allergic patients and should not be used in untreated patients. ${ }^{20}$ CRD was used as a gold standard similar to a previous study that showed that rApi m 1, rApi m 10, rVes v 1, and rVes v 5 facilitated identification of the culprit venom with good agreement with skin testing. ${ }^{21}$ Identification of the causative insect improved with additional CRDs. However, severity of sting reaction was not associated with results obtained by skin testing, venom-specific IgE levels, or molecular diagnosis. ${ }^{5,21}$ Venom skin test responses are negative in many patients with a history of systemic allergic reactions to insect stings, and may be associated with positive serologic test responses to venom-specific
IgE antibodies. Venom skin test responses should be repeated when negative along with a serologic IgE antivenom test, and better diagnostic skin test reagents are urgently needed. ${ }^{22}$

The concentrations of local and commercial allergen venom extracts of honey bee and wasp were the same at $100 \mathrm{mcg} /$ $\mathrm{ml}$ each. The concentration of fire ant used in the local extract was 10 times higher than the concentration in the commercial extract $(1: 10$ vs. 1:100 w/v) in order to get the similar positive result in pilot study. Skin test with both concentrations showed no significant side effects. This might be due to differences in the fire ant species used in extract preparation. The higher concentration of local fire ant allergen extract might explain the higher number positive skin tests and higher sensitivity of local fire ant allergen extract over the commercial version.

In this study, sIgE, CRD, and CCD were considered positive when levels were $\geq 0.35 \mathrm{kUA} / \mathrm{L}$, which is consistent with the internationally accepted cut-off level of $0.35 \mathrm{kU} / \mathrm{l}$ for detecting sIgE. The analytical sensitivity of modern assays is 0.10 $\mathrm{kU} / \mathrm{l}^{23}$ due to the finding that venom sIgE within the range of 0.10 to $0.35 \mathrm{kU} / 1$ may be clinically relevant in patients with low total IgE, and this must be evaluated in the context of the patient's history. However, the level $0.10 \mathrm{kU} / \mathrm{l}$ showed lower specificity, and more false-positive results. The limitation of our study is related to CRD or sIgE-CCD were used as a reference standard test, instead of the sIgE alone because of the potential cross-reactivity between insects across the CCD, and commercially unavailable of the CRD to fire ant.

Future study should be conducted to compare of types of causative insects in nearby regions, cross-reactivity among species of insects, the major allergens in each insect allergen extract, and their role in causing insect allergy.

\section{Conclusions}

SPT and ID with insect venoms could help in confirming and/or identifying the causative insects with the sensitivity of $<75 \%$, except for fire ant $87 \%$, and specificity $<70 \%$. Compare between local and commercial extracts, local fire ant whole body extract showed higher sensitivity, whereas for honey bee, and yellowjacket, commecial extracts showed higher sensitivity.

\section{Acknowledgments}

The authors gratefully acknowledge Mrs. Chaweewan Sripramong for data collection, and Ms. Julaporn Pooliam for assistance with statistical analysis. This study was supported by a Chalermprakiat Grant from the Faculty of Medicine Siriraj Hospital, Mahidol University, and by an NSTDA Chair Professor Grant (P-1450624) funded by the Crown Property Bureau.

\section{Conflict of interest declaration}

The authors declare no conflicts of interest.

\section{Clinical trial registration}

ClinicalTrials.gov NCT03645291 


\section{References}

1. Lertnawapan R, Maek-a-nantawat W. Anaphylaxis and biphasic phase in Thailand: 4-year observation. Allergol Int. 2011;60:283-9.

2. Jirapongsananuruk O, Bunsawansong W, Piyaphanee N, Visitsunthorn N, Thongngarm T, Vichyanond P. Features of patients with anaphylaxis admitted to a university hospital. Ann Allergy Asthma Immunol. 2007;98: 157-62.

3. Bilo BM, Rueff F, Mosbech H, Bonifazi F, Oude-Elberink JN. Diagnosis of Hymenoptera venom allergy. Allergy. 2005;60:1339-49.

4. Golden DB, Demain J, Freeman T, Graft D, Tankersley M, Tracy J, et al. Stinging insect hypersensitivity: A practice parameter update 2016. Ann Allergy Asthma Immunol. 2017;118:28-54.

5. Jakob T, Rafei-Shamsabadi D, Spillner E, Muller S. Diagnostics in Hymenoptera venom allergy: current concepts and developments with special focus on molecular allergy diagnostics. Allergo J Int. 2017;26: 93-105.

6. Golden DB, Tracy JM, Freeman TM, Hoffman DR. Negative venom skin test results in patients with histories of systemic reaction to a sting. J Allergy Clin Immunol. 2003;112:495-8.

7. Rungsa P, Incamnoi P, Sukprasert S, Uawonggul N, Klaynongsruang S, Daduang J, et al. Comparative proteomic analysis of two wasps venom, Vespa tropica and Vespa affinis. Toxicon. 2016;119:159-67.

8. Potiwat R, Tanyaratsrisakul S, Maneewatchararangsri S, Manuyakorn W, Rerkpattanapipat T, Samung Y, et al. Solenopsis geminata (tropical fire ant) anaphylaxis among Thai patients: its allergens and specific IgE-reactivity. Asian Pac J Allergy Immunol. 2018;36:101-8.

9. Paar J, Oldroyd BP, Huettinger E, Kastberger G. Genetic structure of an Apis dorsata population: the significance of migration and colony aggregation. J Hered. 2004;95:119-26.

10. Simons FE, Ardusso LR, Bilo MB, Dimov V, Ebisawa M, El-Gamal YM, et al. 2012 Update: World Allergy Organization Guidelines for the assessment and management of anaphylaxis. Curr Opin Allergy Clin Immunol. 2012;12:389-99.

11. Hunt KJ, Valentine MD, Sobotka AK, Lichtenstein LM. Diagnosis of allergy to stinging insects by skin testing with Hymenoptera venoms. Ann Intern Med. 1976;85:56-9.
12. Valent P, Akin C, Metcalfe DD. Mastocytosis: 2016 updated WHO classification and novel emerging treatment concepts. Blood. 2017;129: 1420-7.

13. Cohen J. A Coefficient of Agreement for Nominal Scales. 1960;20:37-46.

14. Aalberse RC, Akkerdaas J, van Ree R. Cross-reactivity of IgE antibodies to allergens. Allergy. 2001;56:478-90.

15. Caruso B, Bonadonna P, Severino MG, Manfredi M, Dama A, Schiappoli $\mathrm{M}$, et al. Evaluation of the IgE cross-reactions among vespid venoms. A possible approach for the choice of immunotherapy. Allergy. 2007;62:561-4.

16. Monsalve RI, Vega A, Marques L, Miranda A, Fernandez J, Soriano V, et al. Component-resolved diagnosis of vespid venom-allergic individuals: phospholipases and antigen $5 \mathrm{~s}$ are necessary to identify Vespula or Polistes sensitization. Allergy. 2012;67:528-36.

17. Rhoades R. Stinging ants. Curr Opin Allergy Clin Immunol. 2001;1:343-8.

18. Cichocka-Jarosz E, Stobiecki M, Brzyski P, Rogatko I, Nittner-Marszalska M, Sztefko K, et al. Simplification of intradermal skin testing in Hymenoptera venom allergic children. Ann Allergy Asthma Immunol. 2017;118:326-32.

19. Golden DB, Marsh DG, Freidhoff LR, Kwiterovich KA, Addison B, Kagey-Sobotka A, et al. Natural history of Hymenoptera venom sensitivity in adults. J Allergy Clin Immunol. 1997;100:760-6.

20. Rueff F, Przybilla B, Muller U, Mosbech H. The sting challenge test in Hymenoptera venom allergy. Position paper of the Subcommittee on Insect Venom Allergy of the European Academy of Allergology and Clinical Immunology. Allergy. 1996;51:216-25.

21. Gattinger P, Lupinek C, Kalogiros L, Silar M, Zidarn M, Korosec P, et al. The culprit insect but not severity of allergic reactions to bee and wasp venom can be determined by molecular diagnosis. PLoS One. 2018;13:e0199250.

22. Golden DB, Kagey-Sobotka A, Norman PS, Hamilton RG, Lichtenstein LM. Insect sting allergy with negative venom skin test responses. J Allergy Clin Immunol. 2001;107:897-901.

23. Hamilton RG. Proficiency survey-based evaluation of clinical total and allergen-specific IgE assay performance. Arch Pathol Lab Med. 2010;134: 975-82. 